\title{
Dabrafenib-Trametinib-Cetuximab Regimen
}

National Cancer Institute

\section{Source}

National Cancer Institute. Dabrafenib-Trametinib-Cetuximab Regimen. NCI Thesaurus.

Code C161593.

A regimen consisting of dabrafenib, trametinib and cetuximab that can be used in the treatment of certain BRAF-mutated colon and rectal cancers. 